\title{
The AdS/CFT Correspondence and Light-Front QCD
}

\author{
Stanley J. Brodsky* \\ Stanford Linear Accelerator Center, Stanford University, Stanford, California 94309, USA \\ E-mail: sjbtheslac.stanford.edu
}

\section{Guy F. de Téramond}

Universidad de Costa Rica, San José, Costa Rica

E-mail: gdteasterix.crnet.cr

\begin{abstract}
We identify an invariant light-front coordinate $\zeta$ which allows the separation of the dynamics of quark and gluon binding from the kinematics of constituent spin and internal orbital angular momentum. The result is a single-variable light-front Schrödinger equation for QCD which determines the eigenspectrum and the light-front wavefunctions of hadrons for general spin and orbital angular momentum. This frame-independent light-front wave equation is equivalent to the equations of motion which describe the propagation of spin- $J$ modes on anti-de Sitter (AdS) space. Light-front holography is a remarkable feature of AdS/CFT: it allows hadronic amplitudes in the AdS fifth dimension to be mapped to frame-independent light-front wavefunctions of hadrons in physical space-time, thus providing a relativistic description of hadrons at the amplitude level. In principle, the model can be systematically improved by diagonalizing the full QCD light-front Hamiltonian on the AdS/QCD basis. Quark and gluon hadronization can be computed at the amplitude level by convoluting the off-shell $T$ matrix calculated from the QCD light-front Hamiltonian with the hadronic light-front wavefunctions. We also note the distinction between static observables such as the probability distributions computed from the square of the light-front wavefunctions versus dynamical observables such as the structure functions and the leading-twist single-spin asymmetries measured in deep inelastic scattering which include the effects of initial and final-state interactions.
\end{abstract}

LIGHT CONE 2008: Relativistic Nuclear and Particle Physics July 7-11 2008, Mulhouse, France

\footnotetext{
*Speaker.

${ }^{\dagger}$ This research was supported by the Department of Energy contract DE-AC02-76SF00515. SLAC-PUB-13428.
} 


\section{Introduction}

One of the most important theoretical tools in atomic physics is the Schrödinger equation, which describes the quantum-mechanical structure of atomic systems at the amplitude level. Lightfront wavefunctions (LFWFs) play a similar role in quantum chromodynamics (QCD), providing a fundamental description of the structure and internal dynamics of hadrons in terms of their constituent quarks and gluons. The natural concept of a wavefunction for relativistic quantum field theories such as QCD is the light-front wavefunction $\psi_{n}\left(x_{i}, \mathbf{k}_{\perp i}, \lambda_{i}\right)$ which specifies the $n$ quark and gluon constituents of a hadron's Fock state as a function of the light-cone fractions $x_{i}=k^{+} / P^{+}=\left(k^{0}+k^{3}\right) /\left(P^{0}+P^{3}\right)$ transverse momenta $\mathbf{k}_{\perp i}$ and spin projections $\lambda_{i}$. The light-front wavefunctions of bound states in QCD are relativistic generalizations of the Schrödinger wavefunctions of atomic physics, but they are determined at fixed light-cone time $\tau=t+z / c$ - the "front form" introduced by Dirac [1] - rather than at fixed ordinary time $t$.

When a flash from a camera illuminates a scene, each object is illuminated along the lightfront of the flash; i.e., at a given $\tau$. In contrast, setting the initial condition using conventional instant time $t$ requires simultaneous scattering of photons on each constituent. Thus it is natural to set boundary conditions at fixed $\tau$ and then evolve the system using the light-front Hamiltonian $P^{-}=P^{0}-P^{3}=i d / d \tau$. The invariant Hamiltonian $H_{L F}=P^{+} P^{-}-\mathbf{P}_{\perp}^{2}$ then has eigenvalues $\mathscr{M}^{2}$ where $\mathscr{M}$ is the physical mass. Its eigenfunctions are the light-front (LF) eigenstates whose Fock state projections define the light-front wavefunctions.

A remarkable feature of LFWFs is the fact that they are frame independent; i.e., the form of the LFWF is independent of the hadron's total momentum $P^{+}=P^{0}+P^{3}$ and $\mathbf{P}_{\perp}$. The light-front formalism for gauge theories in light-cone gauge $A^{+}=0$ is particularly useful in that there are no ghosts and the gluon polarization is purely transverse: $S_{g}^{z}= \pm 1$. Thus one has a direct physical interpretation of orbital angular momentum. The constituent spin and orbital angular momentum properties of the hadrons are also encoded in the LFWFs. For example, the internal spin and orbital angular momentum is conserved for each $n$-particle LF Fock state: $\sum_{i=1}^{n} S_{i}^{z}+\sum_{i=1}^{n-1} L_{i}^{z}=J^{z}$, since there are $n-1$ relative orbital angular momentum. Since the plus momenta are conserved and positive, the vacuum in front form is trivial except for $k^{+}$zero modes. For example, in the case of the Higgs theory, a $c$-number LF zero mode constant [2] replaces the vacuum condensate of the instant form. The simple structure of the light-front vacuum allows an unambiguous definition of the partonic content of a hadron.

Light-front wavefunctions are the fundamental process-independent amplitudes which encode hadron properties in terms of their quark and gluon degrees of freedom, predicting dynamical quantities such as spin correlations, form factors, structure functions, generalized parton distributions, and exclusive scattering amplitudes. Meson and baryon light-front wavefunctions can be measured in diffractive di-jet and tri-jet reactions, respectively. One of the most important advantages of the light-front formalism is that spacelike form factors can be represented as simple overlap integrals of the LF Fock state wavefunctions $\psi_{n}$ and $\psi_{n^{\prime}}$ with $n^{\prime}=n$; i.e., the Drell-Yan-West formula. This is in dramatic contrast to the usual instant form result which requires the inclusion of contributions where the current couples to vacuum processes. Thus knowing the wavefunction of a hadron at fixed time $t$ is not sufficient to determine the form factors and other properties of the hadron. In addition, one must also be able to compute the boosted instant form wavefunction, which requires 
solving a complex dynamical problem. In fact, boosted wavefunctions are only known at weak coupling and even then are more complicated than the product of Melosh or Wigner transformations of the individual constituent spinors. In contrast, the light-front wavefunctions of a hadron are independent of the momentum of the hadron, and they are thus boost invariant. The generalized parton distributions measured in deep inelastic Compton scattering $\gamma^{*}(q) p \rightarrow \gamma(k) p^{\prime}$ in the handbag approximation can be written as the overlap of light-front wavefunctions [3].

\section{A Single-Variable Light-Front Schrödinger Equation for QCD [4]}

A key step in the analysis of an atomic system such as positronium is the introduction of the spherical coordinates $r, \theta, \phi$ which separates the dynamics of Coulomb binding from the kinematical effects of the quantized orbital angular momentum $L$. The essential dynamics of the atom is specified by the radial Schrödinger equation whose eigensolutions $\psi_{n, L}(r)$ determine the boundstate wavefunction and eigenspectrum. Here we show that there is an analogous invariant light-front coordinate $\zeta$ which allows one to separate the essential dynamics of quark and gluon binding from the kinematical physics of constituent spin and internal orbital angular momentum. The result is a single-variable light-front Schrödinger equation for QCD which determines the eigenspectrum and the light-front wavefunctions of hadrons for general spin and orbital angular momentum. Conversely, this analysis can be applied to atomic physics, providing an elegant formalism for relativistic atoms.

The connection between light-front QCD and the description of hadronic modes on AdS space is physically compelling and phenomenologically successful. To a first approximation light-front QCD is formally equivalent to an effective gravity theory on $\mathrm{AdS}_{5}$. To prove this, we show that the LF Hamiltonian equations of motion of QCD lead to an effective LF wave equation for physical modes $\phi(\zeta)$ which encode the hadronic properties. This LF wave equations carry the orbital angular momentum quantum numbers and are equivalent to the equations of motion which describe the propagation of spin- $J$ modes on AdS space. This allows us to formally establish a gauge/gravity correspondence between an effective gravity theory defined on $\mathrm{AdS}_{5}$ and light front QCD at its asymptotic boundary.

To simplify the discussion we will consider a two-parton hadronic bound state. In the case of massless constituents the LF Hamiltonian equation of motion of QCD leads to the equation

$$
\begin{aligned}
\mathscr{M}^{2} & =\int_{0}^{1} d x \int \frac{d^{2} \mathbf{k}_{\perp}}{16 \pi^{3}} \frac{\mathbf{k}_{\perp}^{2}}{x(1-x)}\left|\psi\left(x, \mathbf{k}_{\perp}\right)\right|^{2}+\text { interactions } \\
& =\int_{0}^{1} \frac{d x}{x(1-x)} \int d^{2} \mathbf{b}_{\perp} \psi^{*}\left(x, \mathbf{b}_{\perp}\right)\left(-\nabla_{\mathbf{b}_{\perp \ell}}^{2}\right) \psi\left(x, \mathbf{b}_{\perp}\right)+\text { interactions. }
\end{aligned}
$$

The functional dependence for a given Fock state is given in terms of the invariant mass $\mathscr{M}_{n}^{2}=$ $\left(\sum_{a=1}^{n} k_{a}^{\mu}\right)^{2}=\sum_{a} \frac{\mathbf{k}_{\perp a}^{2}}{x_{a}} \rightarrow \frac{\mathbf{k}_{\perp}^{2}}{x(1-x)}$, the measure of the off-mass shell energy $\mathscr{M}^{2}-\mathscr{M}_{n}^{2}$. Similarly in impact space the relevant variable for a two-parton state is $\zeta^{2}=x(1-x) \mathbf{b}_{\perp}^{2}$. Thus, to first approximation LF dynamics depend only on the boost invariant variable $\mathscr{M}_{n}$ or $\zeta$ and hadronic properties are encoded in the hadronic mode $\phi(\zeta): \psi\left(x, \mathbf{k}_{\perp}\right) \rightarrow \phi(\zeta)$. We choose the normalization of the LF mode $\phi(\zeta)=\langle\zeta \mid \phi\rangle$ with $\langle\phi \mid \phi\rangle=\int d \zeta|\langle\zeta \mid \phi\rangle|^{2}=1$. Comparing with the LFWF normalization, we 
find the functional relation: $\frac{|\phi|^{2}}{\zeta}=\frac{2 \pi}{x(1-x)}\left|\psi\left(x, \mathbf{b}_{\perp}\right)\right|^{2}$, which is the same result found in [5, 6] from the mapping of transition matrix elements for arbitrary values of the momentum transfer.

We can write the Laplacian operator in circular cylindrical coordinates $\zeta=(\vec{\zeta}, \varphi)$ with $\vec{\zeta}=$ $\sqrt{x(1-x)} \mathbf{b}_{\perp}: \nabla^{2}=\frac{1}{\zeta} \frac{d}{d \zeta}\left(\zeta \frac{d}{d \zeta}\right)+\frac{1}{\zeta^{2}} \frac{\partial^{2}}{\partial \varphi^{2}}$, and factor out the angular dependence of the modes in terms of the $S O(2)$ Casimir representation $L^{2}$ of orbital angular momentum in the transverse plane: $\phi(\vec{\zeta}, \varphi) \sim e^{ \pm i L \varphi} \phi(\zeta)$. We find

$$
\begin{aligned}
\mathscr{M}^{2} & =\int d \zeta \phi^{*}(\zeta) \sqrt{\zeta}\left(-\frac{d^{2}}{d \zeta^{2}}-\frac{1}{\zeta} \frac{d}{d \zeta}+\frac{L^{2}}{\zeta^{2}}\right) \frac{\phi(\zeta)}{\sqrt{\zeta}}+\int d \zeta \phi^{*}(\zeta) U(\zeta) \phi(\zeta) \\
& =\int d \zeta \phi^{*}(\zeta)\left(-\frac{d^{2}}{d \zeta^{2}}-\frac{1-4 L^{2}}{4 \zeta^{2}}+U(\zeta)\right) \phi(\zeta),
\end{aligned}
$$

where all the complexity of the interaction terms in the QCD Lagrangian is summed up in the effective potential $U(\zeta)$. The light-front eigenvalue equation $H_{L F}|\phi\rangle=\mathscr{M}^{2}|\phi\rangle$ is thus a light-front wave equation for $\phi$

$$
\left(-\frac{d^{2}}{d \zeta^{2}}-\frac{1-4 L^{2}}{4 \zeta^{2}}+U(\zeta)\right) \phi(\zeta)=\mathscr{M}^{2} \phi(\zeta)
$$

an effective single-variable light-front Schrödinger equation which is relativistic, covariant and analytically tractable. One can readily generalize the equations to allow for the kinetic energy of massive quarks [7].

As the simplest example we consider a bag-like model [8] where the partons are free inside the hadron and the interaction terms will effectively build confinement. The effective potential is a hard wall: $U(\zeta)=0$ if $\zeta \leq \frac{1}{\Lambda_{\mathrm{QCD}}}$ and $U(\zeta)=\infty$ if $\zeta>\frac{1}{\Lambda_{\mathrm{QCD}}}$. However, unlike the standard bag model [8], boundary conditions are imposed on the boost-invariant variable $\zeta$, not on the bag radius at fixed time. If $L^{2} \geq 0$ the LF Hamiltonian is positive definite $\left\langle\phi\left|H_{L F}\right| \phi\right\rangle \geq 0$ and thus $\mathscr{M}^{2} \geq 0$. If $L^{2}<0$ the LF Hamiltonian is unbounded from below and the particle "falls towards the center". The critical value corresponds to $L=0$. The mode spectrum follows from the boundary conditions $\phi\left(\zeta=1 / \Lambda_{\mathrm{QCD}}\right)=0$, and is given in terms of the roots of Bessel functions: $\mathscr{M}_{L, k}^{2}=\beta_{L, k} \Lambda_{\mathrm{QCD}}$. Since in the conformal limit $U(\zeta) \rightarrow 0$, Eq. (2.3) is equivalent to an AdS wave equation, the hard-wall LF model discussed here is equivalent to the hard wall model of Ref. [9]. Likewise a two-dimensional transverse oscillator with effective potential $U(\zeta) \sim \zeta^{2}$ is equivalent to the softwall model of Ref. [10] which reproduce the usual linear Regge trajectories.

\section{Light-Front Holography}

Our analysis follows from recent developments in light-front QCD [4, 5, 6, 7] which have been inspired by the AdS/CFT correspondence [11] between string states in anti-de Sitter (AdS) space and conformal field theories (CFT) in physical space-time. The application of AdS space and conformal methods to QCD can be motivated from the empirical evidence [12] and theoretical arguments [13] that the QCD coupling $\alpha_{s}\left(Q^{2}\right)$ has an infrared fixed point at low $Q^{2}$. The AdS/CFT correspondence has led to insights into the confining dynamics of QCD and the analytic form of hadronic light-front wavefunctions. As we have shown recently, there is a remarkable mapping between the description of hadronic modes in AdS space and the Hamiltonian formulation of QCD 


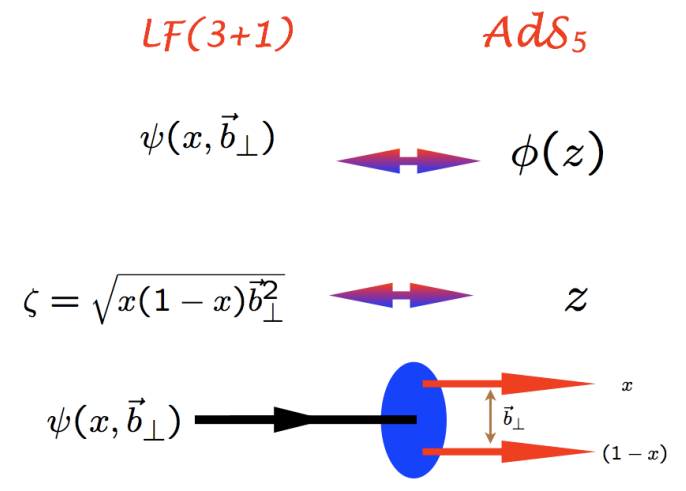

Figure 1: Light-front holography for meson wavefunctions: $\psi\left(x, \mathbf{b}_{\perp}\right)=\sqrt{\frac{x(1-x)}{2 \pi \zeta}} \phi(\zeta)$. This mapping is derived from the equality of the LF and AdS formulae for current matrix elements.

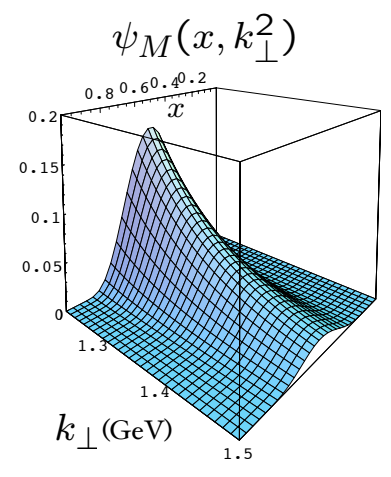

(a)

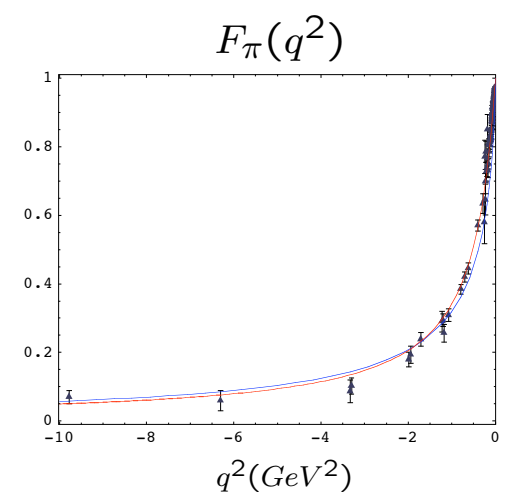

(b)

Figure 2: (a) Pion light-front wavefunction $\psi_{\pi}\left(x, \mathbf{b}_{\perp}\right)$ for the AdS/QCD soft wall $(\kappa=0.375 \mathrm{GeV})$ model. (b) Holographic prediction the space-like pion form factor: (blue) hard wall $\left(\Lambda_{Q C D}=0.32 \mathrm{GeV}\right)$ and (red) soft wall $(\kappa=0.375 \mathrm{GeV})$ models.

in physical space-time quantized on the light-front. This procedure allows string modes $\Phi(z)$ in the AdS holographic variable $z$ to be precisely mapped to the light-front wave functions of hadrons in physical space-time in terms of a specific light-front variable $\zeta$ which measures the separation of the quark and gluonic constituents within the hadron (see fig. 1). The coordinate $\zeta$ also specifies the light-front kinetic energy and invariant mass of constituents. This mapping was originally obtained by matching the expression for electromagnetic current matrix elements in AdS space with the corresponding expression for the current matrix element using light-front theory in physical space time [5, 6]. More recently we have shown that one obtains the identical holographic mapping using the matrix elements of the energy-momentum tensor [14], thus providing an important consistency test and verification of holographic mapping from AdS to physical observables defined on the light front. The resulting wavefunction [see fig. 2 (a)] displays confinement at large interquark separation and conformal symmetry at short distances, reproducing dimensional counting rules for hard exclusive amplitudes. The predictions for the spacelike pion form factor for the hard-wall and soft-wall models is shown in fig. 2 (b).

The use of the invariant coordinate $\zeta$ in light-front QCD allows the separation of the dynamics 
of quark and gluon binding from the kinematics of constituent spin and internal orbital angular momentum. The result is a single-variable light-front Schrödinger equation which determines the eigenspectrum and the light-front wavefunctions of hadrons for general spin and orbital angular momentum. This light-front wave equation serves as a first approximation to QCD and is equivalent to the equations of motion which describe the propagation of spin- $J$ modes on anti-de Sitter (AdS) space. Remarkably the AdS equations correspond to the kinetic energy terms of the partons inside a hadron, whereas the interaction terms build confinement and correspond to the truncation of AdS space [4]. As in this approximation there are no interactions up to the confining scale, there are no anomalous dimensions. This may explain the experimental success of power-law scaling in hard exclusive reactions where there are no indication of the effects of anomalous dimensions. For the same reason we also expect little effect of anomalous dimensions on the gravity side for $J>2$. This also explains why physical hadrons lying on Regge trajectories with $J>2$ are not incompatible with a string description. In the hard wall model there is a total decoupling of the internal orbital angular momentum from the total hadronic spin $J$, and thus the light-front excitation spectrum of hadrons depend only on the orbital and principal quantum numbers. In the hard-wall holographic model the dependence is linear: $\mathscr{M}_{n} \sim 2 n+L$. In the soft-wall model the usual Regge behavior is found $\mathscr{M}^{2} \sim$ $n+L$. One can systematically improve the AdS/QCD approximation by diagonalizing the QCD LF Hamiltonian on the AdS/QCD basis or by generalizing the variational and other systematic methods used in chemistry and nuclear physics. The action of the non-diagonal terms in the QCD interaction Hamiltonian generates the form of the higher Fock state structure of hadronic LFWFs. We emphasize, that in contrast with the original AdS/CFT correspondence, the large $N_{C}$ limit is not required to connect light-front QCD to an effective dual gravity approximation.

\section{Hadronization at the Amplitude Level}

The conversion of quark and gluon partons is usually discussed in terms of on-shell hardscattering cross sections convoluted with ad hoc probability distributions. The LF Hamiltonian formulation of quantum field theory provides a natural formalism to compute hadronization at the amplitude level. In this case one uses light-front time-ordered perturbation theory for the QCD light-front Hamiltonian to generate the off-shell quark and gluon T-matrix helicity amplitude using the LF generalization of the Lippmann-Schwinger formalism:

$$
T^{L F}=H_{I}^{L F}+H_{I}^{L F} \frac{1}{\mathscr{M}_{\text {Initial }}^{2}-\mathscr{M}_{\text {intermediate }}^{2}+i \varepsilon} H_{I}^{L F}+\cdots
$$

Here $\mathscr{M}_{\text {intermediate }}^{2}=\sum_{i=1}^{N}\left(\mathbf{k}_{\perp i}^{2}+m_{i}^{2}\right) / x_{i}$ is the invariant mass squared of the intermediate state and $H_{I}^{L F}$ is the set of interactions of the QCD LF Hamiltonian in the ghost-free light-cone gauge [15]. The $T^{L F}$-matrix element is evaluated between the out and in eigenstates of $H_{L F}^{Q C D}$. The event amplitude generator is illustrated for $e^{+} e^{-} \rightarrow \gamma^{*} \rightarrow X$ in fig. 3. The LFWFS of AdS/QCD can be used as the interpolating amplitudes between the off-shell quark and gluons and the bound-state hadrons. Specifically, if at any stage a set of color-singlet partons has light-front kinetic energy $\sum_{i} \mathbf{k}_{\perp i}^{2} / x_{i}<\Lambda_{Q C D}^{2}$, then one coalesces the virtual partons into a hadron state using the AdS/QCD LFWFs. This provides a specific scheme for determining the factorization scale which matches perturbative and nonperturbative physics. 


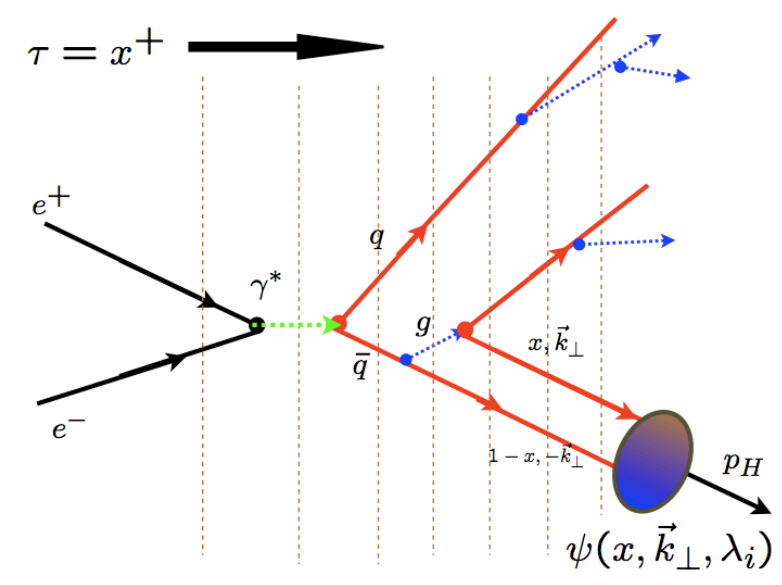

Figure 3: Illustration of an event amplitude generator for $e^{+} e^{-} \rightarrow \gamma^{*} \rightarrow X$ for hadronization processes at the amplitude level. Capture occurs if $\zeta^{2}=x(1-x) \mathbf{b}_{\perp}^{2}>1 / \Lambda_{\mathrm{QCD}}^{2}$ in the AdS/QCD hard wall model of confinement; i.e. if $\mathscr{M}^{2}=\frac{\mathbf{k}_{\perp}^{2}}{x(1-x)}<\Lambda_{\mathrm{QCD}}^{2}$.

This scheme has a number of important computational advantages: (a) Since propagation in LF Hamiltonian theory only proceeds as $\tau$ increases, all particles propagate as forward-moving partons with $k_{i}^{+} \geq 0$. There are thus relatively few contributing $\tau$-ordered diagrams. (b) The computer implementation can be highly efficient: an amplitude of order $g^{n}$ for a given process only needs to be computed once. (c) Each amplitude can be renormalized using the "alternate denominator" counterterm method [16], rendering all amplitudes UV finite. (d) The renormalization scale in a given renormalization scheme can be determined for each skeleton graph even if there are multiple physical scales. (e) The $T^{L F}$-matrix computation allows for the effects of initial and final state interactions of the active and spectator partons. This allows for leading-twist phenomena such as diffractive DIS, the Sivers spin asymmetry and the breakdown of the PQCD Lam-Tung relation in Drell-Yan processes. (f) ERBL and DGLAP evolution are naturally incorporated, including the quenching of DGLAP evolution at large $x_{i}$ where the partons are far off-shell. (g) Color confinement can be incorporated at every stage by limiting the maximum wavelength of the propagating quark and gluons [13].

\section{Conclusions}

We have identified an invariant light-front coordinate $\zeta$ which allows the separation of the dynamics of quark and gluon binding from the kinematics of constituent spin and internal orbital angular momentum. The result is a single-variable light-front Schrödinger equation for QCD which determines the eigenspectrum and the light-front wavefunctions of hadrons for general spin and orbital angular momentum. This frame-independent light-front wave equation is equivalent to the equations of motion which describe the propagation of spin- $J$ modes on anti-de Sitter (AdS) space [4]. Light-Front Holography is one of the most remarkable features of AdS/CFT. It allows one to project the functional dependence of the wavefunction $\Phi(z)$ computed in the AdS fifth dimension to the hadronic frame-independent light-front wavefunction $\psi\left(x_{i}, \mathbf{b}_{\perp i}\right)$ in $3+1$ physical space-time. The variable $z$ maps to $\zeta\left(x_{i}, \mathbf{b}_{\perp i}\right)$. To prove this, we have shown that there exists a 
correspondence between the matrix elements of the energy-momentum tensor of the fundamental hadronic constituents in QCD with the transition amplitudes describing the interaction of string modes in anti-de Sitter space with an external graviton field which propagates in the AdS interior [14]. The agreement of the results for both electromagnetic and gravitational hadronic transition amplitudes provides an important consistency test and verification of holographic mapping from AdS to physical observables defined on the light-front. The transverse coordinate $\zeta$ is related to the invariant mass squared of the constituents in the LFWF and its off-shellness in the light-front kinetic energy, and it is thus the natural variable to characterize the hadronic wavefunction.

It is interesting to note that the form of the nonperturbative pion distribution amplitude $\phi_{\pi}(x)$ obtained from integrating the $q \bar{q}$ valence LFWF $\psi\left(x, \mathbf{k}_{\perp}\right)$ over $\mathbf{k}_{\perp}$, has a quite different $x$-behavior than the asymptotic distribution amplitude predicted from the PQCD evolution [17] of the pion distribution amplitude. The AdS prediction $\phi_{\pi}(x)=\sqrt{3} f_{\pi} \sqrt{x(1-x)}$ has a broader distribution than expected from solving the ERBL evolution equation in perturbative QCD. This observation appears to be consistent with the results of the Fermilab diffractive dijet experiment [18], the moments obtained from lattice QCD [7] and pion form factor data [19].

Nonzero quark masses are naturally incorporated into the AdS predictions [7] by including them explicitly in the LF kinetic energy $\sum_{i}\left(\mathbf{k}_{\perp i}^{2}+m_{i}^{2}\right) / x_{i}$. Given the nonpertubative LFWFs one can predict many interesting phenomenological quantities such as heavy quark decays, generalized parton distributions and parton structure functions. The AdS/QCD model is semiclassical and thus only predicts the lowest valence Fock state structure of the hadron LFWF. In principle, the model can be systematically improved by diagonalizing the full QCD light-front Hamiltonian on the AdS/QCD basis.

Color confinement and its implementation in AdS/QCD implies a maximal wavelength for confined quarks and gluons and thus a finite IR fixed point for the QCD coupling [13]. This strengthens our understanding of the narrow widths of the $J / \psi$ and $\Upsilon$. A new perspective on the nature of quark and gluon condensates in quantum chromodynamics is presented in [20]: the spatial support of QCD condensates is restricted to the interior of hadrons, since they arise due to the interactions of confined quarks and gluons. Chiral symmetry is thus broken in a limited domain of size $1 / m_{\pi}$, in analogy to the limited physical extent of superconductor phases. This picture explains recent results which find no significant signal for the vacuum gluon condensate.

We also note the importance of distinguishing between static observables such as the probability distributions computed from the square of the light-front wavefunctions versus dynamical observables such as the structure functions and the leading twist single-spin asymmetries measured in deep inelastic scattering which include the effects of final state interactions. This distinction is summarized in fig. 4.

\section{Acknowledgments}

Presented by SJB at Light Cone 2008: Relativistic Nuclear And Particle Physics (LC2008) 7-11 Jul 2008, Mulhouse, France. He thanks the Institute for Particle Physics Phenomenology, Durham, UK for its hospitality. We also thank Robert Shrock and James Vary for helpful discussions. 


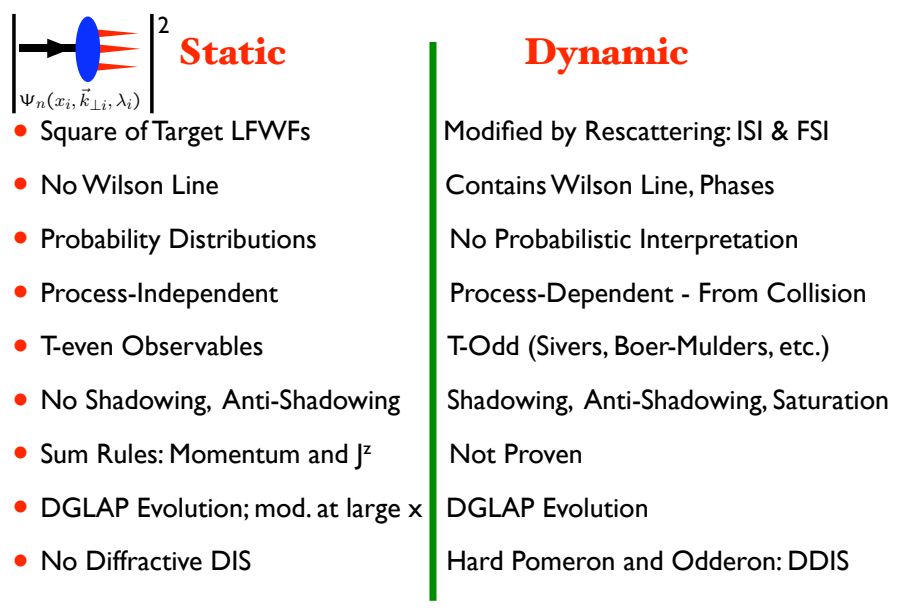

Figure 4: Dynamic versus static observables.

\section{References}

[1] P. A. M. Dirac, Rev. Mod. Phys. 21, 392 (1949).

[2] P. P. Srivastava and S. J. Brodsky, Phys. Rev. D 66, 045019 (2002) [arXiv:hep-ph/0202141].

[3] S. J. Brodsky, M. Diehl and D. S. Hwang, Nucl. Phys. B 596, 99 (2001) [arXiv:hep-ph/0009254].

[4] G. F. de Teramond and S. J. Brodsky, arXiv:0809.4899 [hep-ph].

[5] S. J. Brodsky and G. F. de Teramond, Phys. Rev. Lett. 96, 201601 (2006) [arXiv:hep-ph/0602252].

[6] S. J. Brodsky and G. F. de Teramond, Phys. Rev. D 77, 056007 (2008) [arXiv:0707.3859 [hep-ph]].

[7] S. J. Brodsky and G. F. de Teramond, arXiv:0802.0514 [hep-ph].

[8] A. Chodos, R. L. Jaffe, K. Johnson, C. B. Thorn and V. F. Weisskopf, Phys. Rev. D 9, 3471 (1974).

[9] J. Polchinski and M. J. Strassler, Phys. Rev. Lett. 88, 031601 (2002) [arXiv:hep-th/0109174].

[10] A. Karch, E. Katz, D. T. Son and M. A. Stephanov, Phys. Rev. D 74, 015005 (2006) [arXiv:hep-ph/0602229].

[11] J. M. Maldacena, Adv. Theor. Math. Phys. 2, 231 (1998) [Int. J. Theor. Phys. 38, 1113 (1999)] [arXiv:hep-th/9711200].

[12] A. Deur, V. Burkert, J. P. Chen and W. Korsch, Phys. Lett. B 665, 349 (2008) [arXiv:0803.4119 [hep-ph]].

[13] S. J. Brodsky and R. Shrock, Phys. Lett. B 666, 95 (2008) [arXiv:0806.1535 [hep-th]].

[14] S. J. Brodsky and G. F. de Teramond, Phys. Rev. D 78, 025032 (2008) [arXiv:0804.0452 [hep-ph]].

[15] S. J. Brodsky, H. C. Pauli and S. S. Pinsky, Phys. Rept. 301, 299 (1998) [arXiv:hep-ph/9705477].

[16] S. J. Brodsky, R. Roskies and R. Suaya, Phys. Rev. D 8, 4574 (1973).

[17] G. P. Lepage and S. J. Brodsky, Phys. Lett. B 87, 359 (1979).

[18] E. M. Aitala et al. [E791 Collaboration], Phys. Rev. Lett. 86, 4768 (2001) [arXiv:hep-ex/0010043].

[19] H. M. Choi and C. R. Ji, Phys. Rev. D 74, 093010 (2006) [arXiv:hep-ph/0608148].

[20] S. J. Brodsky and R. Shrock, arXiv:0803.2541 [hep-th]. 